\title{
Principles of non-local field theories and their application to polymerized membranes
}

\author{
Kay Jörg Wiese* \\ Fachbereich Physik, Universität Essen, 45117 Essen, Germany \\ E-mail: wiese@theo-phys.uni-essen.de
}

November 6, 2018

\begin{abstract}
In these lecture notes, we give an overview about non-local field-theories and their application to polymerized membranes, i.e. membranes with a fixed internal connectivity. The main technical tool is the multi-local operator product expansion (MOPE), generalizing ideas from local field theories to the multi-local situation.

These notes are largely inspired by: Kay Wiese, "Polymerized membranes, a review", Domb and Lebowitz, eds., Academic Press, London (2000).

Lectures held for the Graduiertenkolleg in Heidelberg, September 25-27.

Dedicated to Hagen Kleinert at the occasion of his 60th birthday.

Published in Fluctuating Paths and Fields, W. Janke, A. Pelster, H.-J. Schmidt and M. Bachmann (Eds.), World Scientific Singapore (2001)
\end{abstract}

\section{Polymerized membranes}

Mmembranes have attracted much interest during the last years, especially due to their relevance for biological systems. Most of the membranes encountered there are fluid. In this lecture I shall concentrate on another fascinating class of membranes, namely polymerized tethered also called solid membranes. These membrane have a fixed and constant internal metric. They are realized in experiments (e.g. the spectrin network of red blood cells $[1,2]$, or sheets of graphite oxide $[3,4])$.

A microscopic model is given by the so-called "spring and bead model" which consists of balls (beads) which are connected by springs and form a regular lattice. The difficulty is to incororate the self-avoidance of the beads, i.e. the fact that beads cannot intersect.

\section{Representation as non-local field-theory}

We start from the continuous model for a $D$-dimensional flexible polymerized membrane introduced in $[5,6]$, and further studied in [25-28]. This model is a simple extension of the well known Edwards' model for continuous chains. The membrane fluctuates in $d$-dimensional space. Points in the membrane are labeled by coordinates $x \in \mathbb{R}^{D}$ and the configuration of the membrane in physical space is described by the field $r: x \in \mathbb{R}^{D} \longrightarrow$ $r(x) \in \mathbb{R}^{d}$, i.e. from now on we note $r$ instead of $\vec{r}$. At high temperatures the free energy for a configuration is given by the (properly scaled) Hamiltonian

$$
\mathcal{H}[r]=\frac{1}{2-D} \int_{x} \frac{1}{2}(\nabla r(x))^{2}+b_{0} \int_{x} \int_{y} \tilde{\delta}^{d}(r(x)-r(y)) .
$$

The integral $\int_{x}$ runs over $D$-dimensional space and $\nabla$ is the usual gradient operator. The normalizations are $\int_{x}:=\frac{1}{S_{D}} \int \mathrm{d}^{D} x$ with $S_{D}=2 \frac{\pi^{D / 2}}{\Gamma(D / 2)}$ and $\tilde{\delta}^{d}(r(x)-r(y))=(4 \pi)^{d / 2} \delta^{d}(r(x)-r(y))$. The latter term is normally used in Fourier-representation $\tilde{\delta}^{d}(r(x)-r(y))=\int_{p} \mathrm{e}^{i p[r(x)-r(y)]}$, where the normalization of $\int_{p}$ is given by $\int_{p}=\pi^{-d / 2} \int \mathrm{d}^{d} p$ to have $\int_{p} \mathrm{e}^{-p^{2} a}=a^{-d / 2}$. All normalizations are chosen in order to simplify the calculations. The first term in the Hamiltonian is a Gaussian elastic energy which is known to describe the

*New adress: ITP, Kohn Hall, UCSB, Santa Barbara, CA 93106-4030, USA; email: wiese@ itp.ucsb.edu 


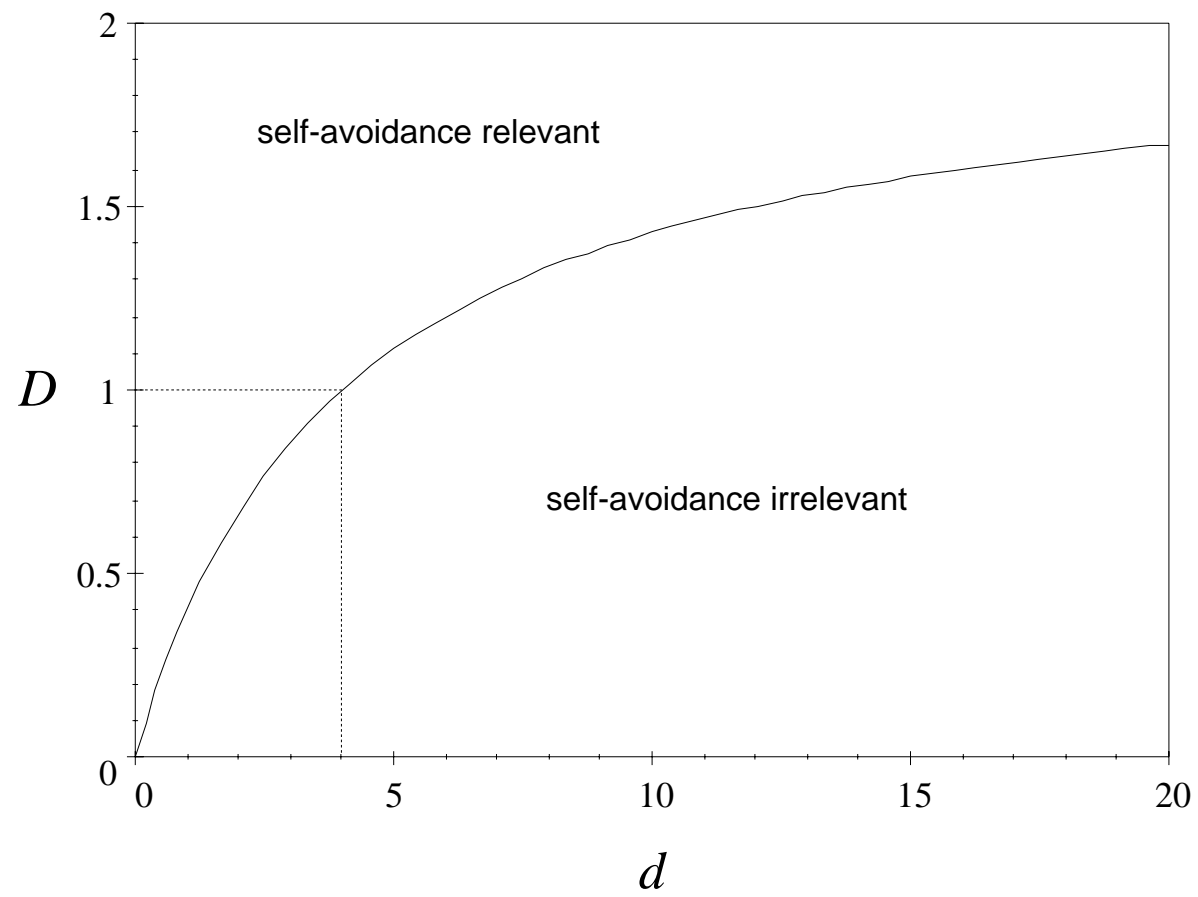

Figure 2.1: The critical curve $\varepsilon(D, d)=0$. The dashed line corresponds to the standard polymer perturbation theory, critical in $d=4$.

free "phantom" surface. The interaction term corresponds (for $b_{0}>0$ ) to a weak repulsive interaction upon contact. The expectation values of physical observables are obtained by performing the average over all fieldconfigurations $r(x)$ with the Boltzmann weight $e^{-\mathcal{H}[r]}$. This average can not be calculated exactly, but one can expand about the configurations of a phantom, i.e. non-interacting surface.

Such a perturbation theory is constructed by performing the series expansion in powers of the coupling constant $b$. This expansion suffers from ultraviolet (UV) divergences which have to be removed by renormalization and which are treated by dimensional regularization, i.e. analytical continuation in $D$ and $d$. Long-range infrared (IR) divergences also appear. They can be eliminated by using a finite membrane, or by studying translationally invariant observables, whose perturbative expansion is also IR-finite in the thermodynamic limit (infinite membrane). Such observables are "neutral" products of vertex operators

$$
\mathcal{O}=\prod_{a=1}^{N} \mathrm{e}^{i k_{a} r\left(x_{a}\right)}, \quad \sum_{a=1}^{N} k_{a}=0 .
$$

Let us now analyze the theory by power-counting. We use internal units $\mu \sim 1 / x$, and note $[x]_{x}=1$, and $[\mu]_{x}=-[\mu]_{\mu}=-1$. The dimension of the field and of the coupling-constant are:

$$
\nu:=[r]_{x}=\frac{2-D}{2}, \quad \varepsilon:=\left[b_{0}\right]_{\mu}=2 D-\nu d .
$$

The interaction is relevant for $\varepsilon>0$, see figure 2.1. Perturbation theory is then expected to be UV-finite except for subtractions associated to relevant operators. We shall come back to this point later.

For clarity, we represent graphically the different interaction terms which have to be considered. The local operators are

$$
1=\mathbf{1}, \quad \frac{1}{2}(\nabla r(x))^{2}=-
$$

The bi-local operator, the dipole, is

$$
\tilde{\delta}^{d}(r(x)-r(y))=\bullet \bullet .
$$

The expectation-value of an observable is

$$
\langle\mathcal{O}[r]\rangle_{b}=\operatorname{Norm} \int \mathcal{D}[r] \mathcal{O}[r] \mathrm{e}^{-\mathcal{H}[r]} .
$$


with Norm chosen such that $\langle\mathbf{1}\rangle_{b}=1$. Perturbatively, all expectation-values are taken with respect to the free theory (again normalized s.t. $\langle\mathbf{1}\rangle_{0}=1$ ):

$$
\langle\mathcal{O}[r]\rangle_{0}=\operatorname{Norm}^{\prime} \int \mathcal{D}[r] \mathcal{O}[r] \mathrm{e}^{-\frac{1}{2-D} \int_{x} \frac{1}{2}(\nabla r(x))^{2}} .
$$

A typical term in the expansion of (2.6) is

$$
\left(-b Z_{b} \mu^{\varepsilon}\right)^{n} \iint \ldots \iint\langle\mathcal{O} \bullet \cdot \ldots \bullet \cdot \bullet\rangle_{0}^{\mathrm{c}},
$$

where the integral runs over the positions of all dipole-endpoints.

\section{Locality of Divergences and the multilocal operator product expansion (MOPE)}

The next step to show ist that all divergences are short distance divergences. Note that even for massless theories and in the absence of IR-divergences, this is not trivial. Divergences could as well appear, when some of the distances involved become equal, or multiple of each other. A simple counter-example is the integral of ||$a \mid-$ $\left.|b|\right|^{-\nu d}$, where $a$ and $b$ are two of the distances involved. Due to a lack of space, we can not give the proof here. The interested reader should consult [7].

As in local field-theoies, divergences can be analyzed via an operator product expansion. Intuitively, in the context of multilocal theories - by which we mean that the interaction depends on more than one point - we also expect multilocal operators to appear in such an operator product expansion, which therefore will be called "multi-local operator product expansion" (MOPE) [8,9]. An example for such an operator product expansion is
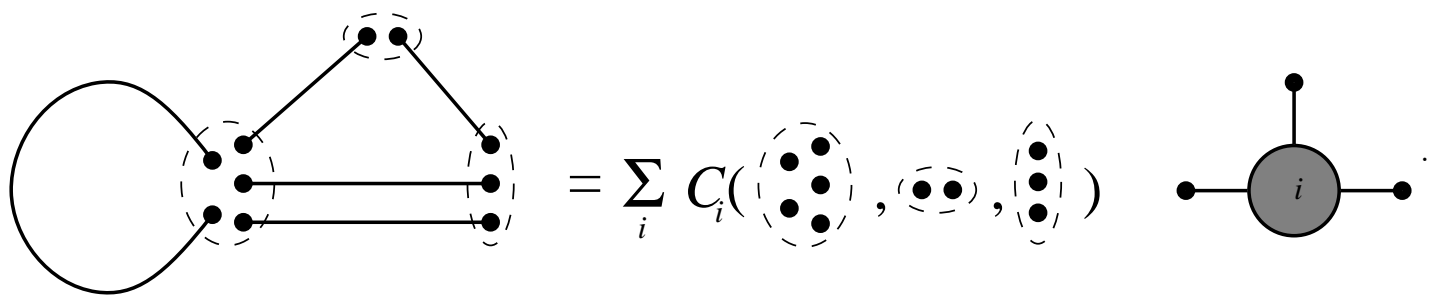

$(3.1)$

Let us explain the formula. We consider $n$ dipoles (here $n=5$ ) and we separate the $2 n$ end-points into $m$ subsets (here $m=3$ ) delimited by the dashed lines. The MOPE describes how the product of these $n$ dipoles behaves when the points inside each of the $m$ subsets are contracted towards a single point $z_{j}$. The result is a sum over multilocal operators $\Phi_{i}\left(z_{1}, \ldots, z_{m}\right)$, depending on the $m$ points $z_{1}, \ldots, z_{m}$, of the form

$$
\sum_{i} C_{i}\left(x_{1}-z_{1}, \ldots\right) \Phi_{i}\left(z_{1}, z_{2}, \ldots, z_{m}\right)
$$

where the MOPE-coefficients $C_{i}\left(x_{1}-z_{1}, \ldots\right)$ depend only on the distances $x_{l}-z_{j}$ inside each subset. This expansion is valid as an operator-identity, i.e. inserted into any expectation value and in the limit of small distances between contracted points. No other operator should appear at the points $z_{1}, \ldots, z_{m}$, towards which the operators are contracted. As the Hamiltonian (2.1) does not contain a mass-scale, the MOPE-coefficients are homogeneous functions of the relative positions between the contracted points, with the degree of homogeneity given by dimensional analysis. In the case considered here, where $n$ dipoles are contracted to an operator $\Phi_{i}$, this degree is $-n \nu d-\left[\Phi_{i}\right]_{x}$. This means that

$$
C_{i}\left(\lambda\left(x_{1}-z_{1}\right), \ldots\right)=\lambda^{-n \frac{2-D}{2} d-\left[\Phi_{i}\right]_{x}} C_{i}\left(x_{1}-z_{1}, \ldots\right),
$$

where $\left[\Phi_{i}\right]_{x}$ is the canonical dimension of the operator $\Phi_{i}$ and $-d(2-D) / 2$ is the canonical dimension of the dipole.

In order to evaluate the associated singularity, one finally has to integrate over all relative distances inside each subset. This gives an additional scale factor with degree $D(2 n-m)$. A singular configuration, such as in Eq. (3.1), will be UV-divergent if this degree of divergence

$$
D(2 n-m)-n \frac{2-D}{2} d-\left[\Phi_{i}\right]_{x},
$$

is negative. It is superficially divergent if the degree is zero and convergent otherwise. The idea of renormalization is to remove exactly these superficially divergent contributions recursively. 


\section{Evaluation of the MOPE-coefficients}

The MOPE gives a convenient and powerful tool to calculate the dominant and all subdominant contributions from singular configurations. In this section, we explain how to calculate the MOPE-coefficients on some explicit examples. These examples will turn out to be the necessary diagrams at 1-loop order.

In the following we shall use the notion of normal-ordering. The first thing, which we use, is that

$$
: \mathrm{e}^{i k r(x)}:=\mathrm{e}^{i k r(x)} \text {. }
$$

Explicitly, tadpole-like contributions which are powers of $\int \mathrm{d}^{D} p \frac{1}{p^{2}}$ are omitted. This is done via a finite part prescription (analytic continuation, dimensional regularization), valid for infinite membranes, for which the normalorder prescription is defined. Let us stress that this is a pure technical trick, which can be cirumvented at the expense of more cumbersome calculations.

The key-formula for all further manipulations is

$$
: \mathrm{e}^{i k r(x)}:: \mathrm{e}^{i p r(y)}:=\mathrm{e}^{k p C(x-y)}: \mathrm{e}^{i k r(x)} \mathrm{e}^{i p r(y)}:
$$

This can be proven as follows: Consider the (free) expectation value of any observable $\mathcal{O}$ times the operators of Eq. (4.2). Then the left- and right-hand sides of the above equation read $\mathcal{L}=\left\langle\mathcal{O}: \mathrm{e}^{i k r(x)}:: \mathrm{e}^{i p r(y)}:\right\rangle_{0}$ and $\mathcal{R}=\mathrm{e}^{k p C(x-y)}\left\langle\mathcal{O}: \mathrm{e}^{i k r(x)} \mathrm{e}^{i p r(y)}:\right\rangle_{0}$. First of all, for $\mathcal{O}=1$, the desired equality of $\mathcal{L}=\mathcal{R}$ holds, because $\left\langle: \mathrm{e}^{i k r(x)} \mathrm{e}^{i p r(y)}:\right\rangle_{0}=1$ and $\left\langle: \mathrm{e}^{i k r(x)}:: \mathrm{e}^{i p r(y)}:\right\rangle_{0}=\mathrm{e}^{k p C(x-y)}$. Now consider a non-trivial observable $\mathcal{O}$, and contract all its fields $r$ with $\mathrm{e}^{i k r(x)}$ or $\mathrm{e}^{i p r(y)}$, before contracting any of the fields $r(x)$ with $r(y)$. The result is a product of correlation-functions between the points in $\mathcal{O}$ and $x$ or $y$, and these are equivalent for both $\mathcal{L}$ and $\mathcal{R}$. However, contracting an arbitrary number of times $\mathrm{e}^{i k r(x)}$, leaves the exponential $\mathrm{e}^{i k r(x)}$ invariant. Completing the contractions for $\mathcal{L}$ therefore yields a factor of $\mathrm{e}^{k p C(x-y)}$, and the latter one also appears in $\mathcal{R}$. Thus, the equality of $\mathcal{L}$ and $\mathcal{R}$ holds for all $\mathcal{O}$ and this proves Eq. (4.2).

Now proceed to the first explicit example, the contraction of a single dipole with endpoints $x$ and $y$ :

$$
\bigcap_{x}=\int_{k}: \mathrm{e}^{i k r(x)}:: \mathrm{e}^{-i k r(y)}: \text {. }
$$

This configuration may have divergences when $x$ and $y$ come close together. Let us stress that in contrast to $\phi^{4}$ theory, these divergences are not obtained as a finite sum of products of correlators: Since $C(x-y)=|x-y|^{2-D}$, the latter is always well-behaved at $x=y$. The singularity only appears when summing an infinite series of diagrams as we will do now. To this purpose, we first normal-order the two exponentials using Eq. (4.2)

$$
\int_{k}: \mathrm{e}^{i k[r(x)-r(y)]}: \mathrm{e}^{-k^{2}|x-y|^{2 \nu}}
$$

Note that the operators $\mathrm{e}^{i k r(x)}$ and $\mathrm{e}^{-i k r(y)}$ are free of divergences upon approaching each other, since no more contractions can be made. The divergence is captured in the factor $\mathrm{e}^{-k^{2}|x-y|^{2 \nu}}$. Therefore, we can expand the exponential : $\mathrm{e}^{i k[r(x)-r(y)]}$ : for small $x-y$ and consequently in powers of $[r(x)-r(y)]$. This expansion is

$$
\int_{k}:\left\{\mathbf{1}+i k[r(x)-r(y)]-\frac{1}{2}(k[r(x)-r(y)])^{2}+\ldots\right\}: \mathrm{e}^{-k^{2}|x-y|^{2 \nu}} .
$$

We truncated the expansion after the third term. It will turn out later that this is sufficient, since subsequent terms in the expansion are proportional to irrelevant operators for which the integral over the MOPE-coefficient is UV-convergent.

Due to the symmetry of the integration over $k$ the term linear in $k$ vanishes. Also due to symmetry, the next term can be simplified with the result

$$
\int_{k}\left[\mathbf{1}-\frac{k^{2}}{2 d}:[r(x)-r(y)]^{2}:+\ldots\right] \mathrm{e}^{-k^{2}|x-y|^{2 \nu}} .
$$

Finally, the integration over $k$ can be performed. Recall that normalizations were chosen such that $\int_{k} \mathrm{e}^{-s k^{2}}=$ $s^{-d / 2}$ to obtain

$$
\Omega=(\bigcap \mid 1) \mathbf{1}+(\bigcap \mid \alpha+\beta) \alpha+\ldots,
$$


where we used the notation ${ }_{\alpha}-\beta=\frac{1}{2}\left(\partial_{\alpha} r\right)\left(\partial_{\beta} r\right)$ and the MOPE-coefficients (reminding Feynman's bra and ket notation)

$$
\begin{aligned}
(0 \mid \mathbf{1}) & =|x-y|^{-\nu d} \\
\left({ }_{\alpha} \mid \beta\right) & =-\frac{1}{2}(x-y)_{\alpha}(x-y)_{\beta}|x-y|^{-\nu(d+2)} .
\end{aligned}
$$

As long as the angular average is taken (and this will be the case when integrating the MOPE-coefficient to obtain the divergence), we can replace in Eq. (4.7) $\alpha-\beta$ by $-=\frac{1}{2}(\nabla r)^{2}$ and Eq. (4.9) by

$$
(\bigcap+-)=-\frac{1}{2 D}|x-y|^{D-\nu d} \text {. }
$$

Next consider a real multi-local example of an operator-product expansion, namely the contraction of two dipoles towards a single dipole:

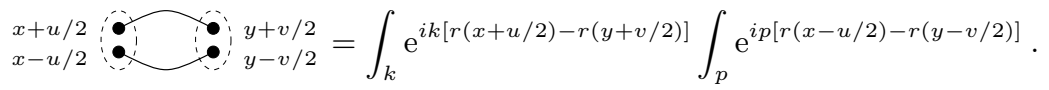

This has to be analyzed for small $u$ and $v$, in order to control the divergences in the latter distances. As above, we normal-order operators which are approached, yielding

$$
\begin{aligned}
\mathrm{e}^{i k r(x+u / 2)} \mathrm{e}^{i p r(x-u / 2)} & =: \mathrm{e}^{i k r(x+u / 2)}:: \mathrm{e}^{i p r(x-u / 2)}: \\
& =: \mathrm{e}^{i k r(x+u / 2)} \mathrm{e}^{i p r(x-u / 2)}: \mathrm{e}^{k p C(u)} .
\end{aligned}
$$

A similar formula holds when approaching $\mathrm{e}^{-i k r(y+v / 2)}$ and $\mathrm{e}^{-i p r(y-v / 2)}$. Eq. (4.11) then becomes

$$
\int_{k} \int_{p}: \mathrm{e}^{i k r(x+u / 2)+i p r(x-u / 2)}:: \mathrm{e}^{-i k r(y+v / 2)-i p r(y-v / 2)}: \mathrm{e}^{k p[C(u)+C(v)]} .
$$

In order to keep things as simple as possible, let us first extract the leading contribution before analyzing subleading corrections. This leading contribution is obtained when expanding the exponential operators (here exemplified for the second one) as

$$
: \mathrm{e}^{-i k r(y+v / 2)} \mathrm{e}^{-i p r(y-v / 2)}:=: \mathrm{e}^{-i(k+p) r(y)}(1+O(\nabla r)):
$$

and dropping terms of order $\nabla r$. This simplifies Eq. (4.13) to

$$
\int_{k} \int_{p}: \mathrm{e}^{i(k+p) r(x)}:: \mathrm{e}^{-i(k+p) r(y)}: \mathrm{e}^{k p[C(u)+C(v)]} .
$$

In the next step, first $k$ and second $p$ are shifted $k \longrightarrow k-p$ then $p \longrightarrow p+\frac{k}{2}$. The result is (dropping the normal-ordering according to Eq. (4.1))

$$
\int_{k} \mathrm{e}^{i k[r(x)-r(y)]} \int_{p} \mathrm{e}^{\left(\frac{1}{4} k^{2}-p^{2}\right)[C(u)+C(v)]} .
$$

The factor of $\int_{k} \mathrm{e}^{i k[r(x)-r(y)]}$ is again a $\delta$-distribution, and the leading term of the short distance expansion of Eq. (4.16). Derivatives of the $\delta$-distribution appear when expanding $\mathrm{e}^{\left(\frac{1}{4} k^{2}-p^{2}\right)[C(u)+C(v)]}$ in $k^{2}$; these are less relevant and only the first sub-leading term will be displayed for illustration:

$$
\begin{aligned}
& \int_{k} \mathrm{e}^{i k[r(x)-r(y)]} \int_{p} \mathrm{e}^{-p^{2}[C(u)+C(v)]}\left(1+\frac{k^{2}}{4}[C(u)+C(v)]+\ldots\right) \\
& =(\bullet \bullet \bullet \bullet \cdot \bullet+(\bullet \bullet \bullet) \cdots+\ldots,
\end{aligned}
$$

where in analogy to Eqs. (4.7) to (4.9)

$$
\begin{aligned}
(\bullet \cdot) & =[C(u)+C(v)]^{-d / 2} \\
\bullet & \bullet
\end{aligned}
$$

with $\bullet \cdot=\tilde{\delta}^{d}(r(x)-r(y))$ and $\bullet \Vdash=\left(-\Delta_{r}\right) \tilde{\delta}^{d}(r(x)-r(y))$. 
Let us already mention that the leading contribution proportional to the $\delta$-distribution will renormalize the coupling-constant, and that the next-to-leading term is irrelevant and can be neglected. The same holds true for the additional term proportional to $(\nabla r)$ which was dropped in Eq. (4.14).

There is one more possible divergent contribution at the 1-loop level, namely .... We now show that the leading term of its expansion, which is expected to be proportional to $\bullet \cdot$, is trivial. To this aim consider

$$
\begin{aligned}
& \text { O. } \\
& =\int_{k, p}: \mathrm{e}^{i k r(u)}:: \mathrm{e}^{-i k r(x)} \mathrm{e}^{i p r(y)} \mathrm{e}^{-i p r(z)}: \mathrm{e}^{-p^{2} C(y-z)} \mathrm{e}^{k p[C(x-z)-C(x-y)]} .
\end{aligned}
$$

We want to study the contraction of $x, y$, and $z$, and look for all contributions which are proportional to $\bullet$ • $=$ $\int_{k}: \mathrm{e}^{i k r(u)}:: \mathrm{e}^{-i k r((x+y+z) / 3)}:$. The key-observation is that in Eq. (4.19) the leading term is obtained by approximating $\mathrm{e}^{k p[C(x-z)-C(x-y)]} \approx 1$. All subsequent terms yield factors of $k$, which after integration over $k$ give derivatives of the $\tilde{\delta}^{d}$-distribution. The result is that

$$
(.0 \mid \cdot \bullet)-(\bigcap \mid 1)=0 \text {. }
$$

This means that divergences of .... are already taken into account by a proper treatment of the divergences in .. , analyzed in Eq. (4.7).

\section{Renormalization at 1-loop order}

In the last sections, we discussed how divergences occur, how their general structure is obtained by the MOPE, and how the MOPE-coefficients are calculated. In the next step, the theory shall be renormalized. The basic idea is to identify the divergences through the MOPE, and then to introduce counter-terms which subtract these divergences. These counter-terms are nothing else than integrals over the MOPE-coefficients, properly regularized, i.e. cut off. We introcuce two renormalization group factors $Z$ (renormalizing the field $r$ ) and $Z_{b}$ (renormalizing the coupling b). Recalling Eq. (2.1), this is

$$
\mathcal{H}[r]=\frac{Z}{2-D} \int_{x} \frac{1}{2}(\nabla r(x))^{2}+b Z_{b} \mu^{\varepsilon} \int_{x} \int_{y} \tilde{\delta}^{d}(r(x)-r(y))
$$

where $r$ and $b$ are the renormalized field and renormalized dimensionless coupling constant, and $\mu=L^{-1}$ is the renormalization momentum scale. (To be precise: The field $r$ in Eq. (2.1) is the bare field and should be noted $\left.r_{0}.\right)$

Let us start to eliminate the divergences in the case, where the end-points $(x, y)$ of a single dipole are contracted towards a point (taken here to be the center-of-mass $z=(x+y) / 2$ ). The MOPE was given in Eq. (4.7) ff. We there have to distinguish between counter-terms for relevant operators and those for marginal operators. The former can be defined by analytic continuation, while the latter require a subtraction scale. Indeed, the divergence proportional to $\mathbf{1}$ is given by the integral

$$
\int_{\Lambda^{-1}<|x-y|<L}\left(\bigcap_{x} \mid \mathbf{1}\right)=\int_{\Lambda^{-1}}^{L} \frac{\mathrm{d} x}{x} x^{D-\nu d}=\frac{1}{D-\varepsilon}\left(\Lambda^{D-\varepsilon}-L^{\varepsilon-D}\right),
$$

where $\Lambda$ is a high-momentum UV-regulator and $L$ a large distance regulator. For $\varepsilon \approx 0$ this is UV-divergent but IR-convergent. The simplest way to subtract this divergence is therefore to replace the dipole operator by

$$
{ }_{x}^{\bullet}{ }_{y} \longrightarrow_{x} \bullet{ }_{y}-{ }_{x}^{\bullet \cdots \cdots \cdots \cdot{ }_{y},}
$$

where ${ }_{x} \cdots \cdots \cdots \cdot{ }_{y}=|x-y|^{-\nu d}$. This amounts to adding to the bare Hamiltonian (2.1) the UV-divergent counterterm

$$
\Delta \mathcal{H}_{1}=-b Z_{b} \mu^{\varepsilon} \int_{x} \int_{y}|x-y|^{-\nu d}
$$

which is a pure number and thus does not change the expectation-value of any physical observable.

We next consider marginal operators: In the MOPE of Eq. (4.9), the integral over the relative distance of $\int_{x-y}\left({ }_{x} \Omega_{y} \mid \alpha+\beta\right) \alpha+\beta$ is logarithmically divergent at $\varepsilon=0$. In order to find the appropriate counter-term, we use dimensional regularization, i.e. set $\varepsilon>0$. An IR-cutoff $L$, or equivalently a subtraction momentum scale 
$\mu=L^{-1}$, has to be introduced in order to define the subtraction operation. As a general rule, let us integrate over all distances appearing in the MOPE-coefficient, bounded by the subtraction scale $L=\mu^{-1}$. Defining

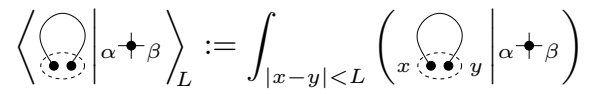

we need the following counter-term in the Hamiltonian

$$
\Delta \mathcal{H}_{+}=-b \mu^{\varepsilon}\left\langle\bigcap_{\alpha+\beta}\right\rangle_{L} \int_{x} \alpha{ }^{-\beta}{ }_{x}
$$

subtracting explicitly the divergence in the integrals. The reader is invited to verify this explicitly on the example of the expectation value of $\mathcal{O}=\mathrm{e}^{i k[r(s)-r(t)]}$. The solution is given in appendix $\mathrm{H}$ of [7].

Since the angular integration in Eq. (5.5) reduces $\alpha+\beta$ to $\downarrow$, we can replace Eq. (5.6) by the equivalent expression

$$
\Delta \mathcal{H}_{+}=-b \mu^{\varepsilon}\langle\bigcirc \mid+\rangle_{L} \int_{x}+x
$$

for which the numerical value of the diagram is calculated as

$$
\langle\bigcirc \mid+\rangle_{L}=\int_{|x-y|<L}\left(\bigcap_{y} \mid+\right)=-\frac{1}{2 D} \int_{0}^{L} \frac{\mathrm{d} x}{x} x^{2 D-\nu d}=-\frac{1}{2 D} \frac{L^{\varepsilon}}{\varepsilon} .
$$

We can now subtract this term in a minimal subtraction scheme (MS). The internal dimension of the membrane $D$ is kept fixed and (5.8) is expanded as a Laurent series in $\varepsilon$, which here starts at $\varepsilon^{-1}$. The residue of the pole in Eq. (5.8) is

$$
\langle\bigcirc \mid+\rangle_{\varepsilon}=-\frac{1}{2 D} \text {. }
$$

It is this pole that is subtracted in the MS-scheme by adding to the Hamiltonian a counter-term

$$
\Delta \mathcal{H}_{+}=-\frac{b}{\varepsilon}\left\langle\bigcap_{+}\right\rangle_{\varepsilon} \int_{x}{ }^{-} x
$$

Similarly, the divergence arising from the contraction of two dipoles to a single dipole is subtracted by a counter-term

$$
\left.\Delta \mathcal{H} \longrightarrow=b^{2} \mu^{2 \varepsilon}: \bullet \cdot\right\rangle_{L} \int_{x} \int_{y} x \bullet{ }_{y}
$$

with

$$
\langle\cdot\rangle_{L}=\int_{|x|<L} \int_{|y|<L}(\bullet: \bullet \cdot \bullet
$$

Reducing this integral counter-term to a number, we subtract the residue of the single pole of

$$
\langle\cdot\rangle_{L}=\int_{|x|<L} \int_{|y|<L}(\bullet \bullet \cdot)=\int_{|x|<L} \int_{|y|<L}\left(|x|^{2 \nu}+|y|^{2 \nu}\right)^{-d / 2}
$$

Note that the regulator $L$ cuts off both integrations. One can now either utilize some simple algebra or show by the methods of conformal mapping (see [7]) that the residue is obtained by fixing one distance to 1 and by freely integrating over the remaining one

$$
\text { : }\rangle_{\varepsilon}=\int_{0}^{\infty} \frac{\mathrm{d} x}{x} x^{D}\left(1+x^{2-D}\right)^{-\frac{2 D}{2-D}}=\frac{1}{2-D} \frac{\Gamma\left(\frac{D}{2-D}\right)^{2}}{\Gamma\left(\frac{2 D}{2-D}\right)} \text {. }
$$

As a result, the model is UV-finite at 1-loop order, if we use in the renormalized Hamiltonian (5.1) the renormalization factors $Z$ and $Z_{b}$

$$
\begin{aligned}
& Z=1-(2-D)\langle\bigcirc \mid \cdot\rangle_{\varepsilon} \frac{b}{\varepsilon}+O\left(b^{2}\right) \\
& Z_{b}=1+\langle\bullet\rangle_{\varepsilon} \frac{b}{\varepsilon}+O\left(b^{2}\right) .
\end{aligned}
$$

Note that due to Eq. (4.19) no counter-term for .... is necessary.

The renormalized field and coupling are re-expressed in terms of their bare counterparts through

$$
r_{0}(x)=Z^{1 / 2} r(x), \quad b_{0}=b Z_{b} Z^{d / 2} \mu^{\varepsilon} .
$$


Finally, the renormalization group functions are obtained from the variation of the coupling constant and the field with respect to the renormalization scale $\mu$, keeping the bare coupling fixed. The flow of the coupling is written in terms of $Z$ and $Z_{b}$ with the diagrams given in Eqs. (5.9) and (5.14) as

$$
\begin{aligned}
\beta(b) & :=\left.\mu \frac{\partial}{\partial \mu}\right|_{b_{0}} b=\frac{-\varepsilon b}{1+b \frac{\partial}{\partial b} \ln Z_{b}+\frac{d}{2} b \frac{\partial}{\partial b} \ln Z} \\
& =-\varepsilon b+\left(\langle\cdot\rangle_{\varepsilon}-\nu d\langle\bigcirc-\rangle_{\varepsilon}\right) b^{2}+O\left(b^{3}\right) .
\end{aligned}
$$

Similarly, the full dimension of the field (the exponent entering into the correlation function) is obtained as

$$
\begin{aligned}
\nu(b) & :=\frac{2-D}{2}-\left.\frac{1}{2} \mu \frac{\partial}{\partial \mu}\right|_{b_{0}} \ln Z=\frac{2-D}{2}-\frac{1}{2} \beta(b) \frac{\partial}{\partial b} \ln Z \\
& =\frac{2-D}{2}\left[1-b\left\langle(-\rangle_{\varepsilon}\right]+O\left(b^{2}\right)=\frac{2-D}{2}\left[1+b \frac{1}{2 D}\right]+O\left(b^{2}\right) .\right.
\end{aligned}
$$

Note that minimal subtraction is used on the level of counter-terms or equivalently $Z$-factors. Since $Z$ enters as $Z^{d}$ into the $\beta$-function, the latter also contains a factor of $d$ in the 1-loop approximation, i.e. $Z^{d}$ is not minimally renormalized. In order to calculate the leading order in $\varepsilon$, the factor of $d$ can be replaced by $d_{c}=\frac{4 D}{2-D}$.

The $\beta$-function has a non-trivial fixed-point with $\beta\left(b^{*}\right)=0$, which has positive slope and thus describes the behavior of the model at large distances. The anomalous dimension $\nu^{*}:=\nu\left(b^{*}\right)$ becomes to first order in $\varepsilon$

$$
\nu^{*}=\frac{2-D}{2}\left[1+\frac{\varepsilon}{2 D}\left(\frac{1}{2-D} \frac{\Gamma\left(\frac{D}{2-D}\right)^{2}}{\Gamma\left(\frac{2 D}{2-D}\right)}+1\right)^{-1}\right]+O\left(\varepsilon^{2}\right) .
$$

For polymers, this result reduces to the well-known formula $\nu^{*}(D=1)=\frac{1}{2}+\frac{4-d}{16}+O\left((4-d)^{2}\right)$.

\section{Results for self-avoiding membranes from 2-loop calculations}

Two loop results are obtained by an explicit analytic and numeric integration of the following combination of diagrams (which are all of order $1 / \varepsilon$ )

$$
\begin{aligned}
& \mathcal{C}_{1}=-\frac{2}{3}\langle\cdot \bullet \cdot \bullet\rangle_{L}+\left(\langle\cdot \bullet\rangle_{L}\right)^{2} \\
& \mathcal{C}_{2}=-2\langle\cdot \bullet\rangle_{L}+\langle\bigcirc \mid \cdot\rangle_{L}\langle\cdot \bullet \cdot \bullet\rangle_{L} \\
& \mathcal{C}_{3}=\langle\bigcirc \mid+\rangle_{\varepsilon^{-1}}\left(\langle\bullet \cdot \bullet\rangle_{L}+\frac{(2-D) d}{4}\langle\cdot \bullet \cdot\rangle_{\varepsilon^{-1}}\right)
\end{aligned}
$$

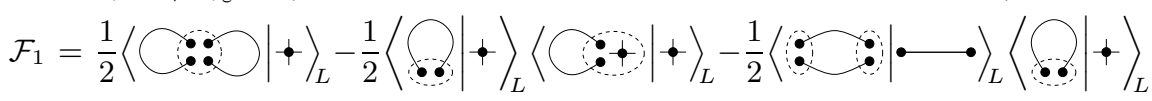

$$
\begin{aligned}
& \mathcal{F}_{2}=-\frac{1}{2}\langle\bigcirc \mid+\rangle_{\varepsilon^{-1}} \times \\
& \times\left(\langle\bigcirc \bullet-+\rangle_{L}+\frac{(2-D)(d+2)}{2}\langle\bigcirc \mid+\rangle_{L}-\langle\bigcirc \mid+\rangle_{L}\langle-+\mid+\rangle_{L}\right) \\
& \mathcal{F}_{3}=\frac{1}{2}\langle\bigcirc \mid \cdot\rangle_{\varepsilon^{-1}}\left(\langle\cdot \bullet\rangle_{L}-\langle\cdot \bullet\rangle_{\varepsilon^{-1}}\right) .
\end{aligned}
$$

One then has the freedom to extrapolate about any point on the critical curve $\varepsilon(D, d)=0$. This freedom - or ambiguity - can be used to optimize the results. The results of such a procedure for the 2-loop cacluations of $\nu^{*}$ are given on figure 6.1 for membranes $(D=2)$ in $d$ dimensions $(2 \leq d \leq 20)$. We see that for $d \rightarrow \infty$ the prediction of a Gaussian variational method $\nu_{\mathrm{var}}=\frac{2 D}{d}$ becomes exact. For small $d$, the prediction made by Flory's argument $\nu_{\text {Flory }}=\frac{2+D}{2+d}$ is close to our results. This is a non-trivial result, since the membrane case corresponds to $\varepsilon=4$ and in comparison with polymers in $d=3$, where $\varepsilon=1 / 2$, the 2-loop corrections were expected to be large. In fact, they are small when one expands around the critical curve $\varepsilon=0$ for an adequate range of $D \sim 1.5$ (depending slightly on $d$ and on the choice of variables) and a suitable choice of extrapolation variables. In this case the 2-loop corrections are even smaller than the 1-loop corrections and allow for more reliable extrapolations to $\varepsilon=4$. This can be understood from the large order behavior [10]. 


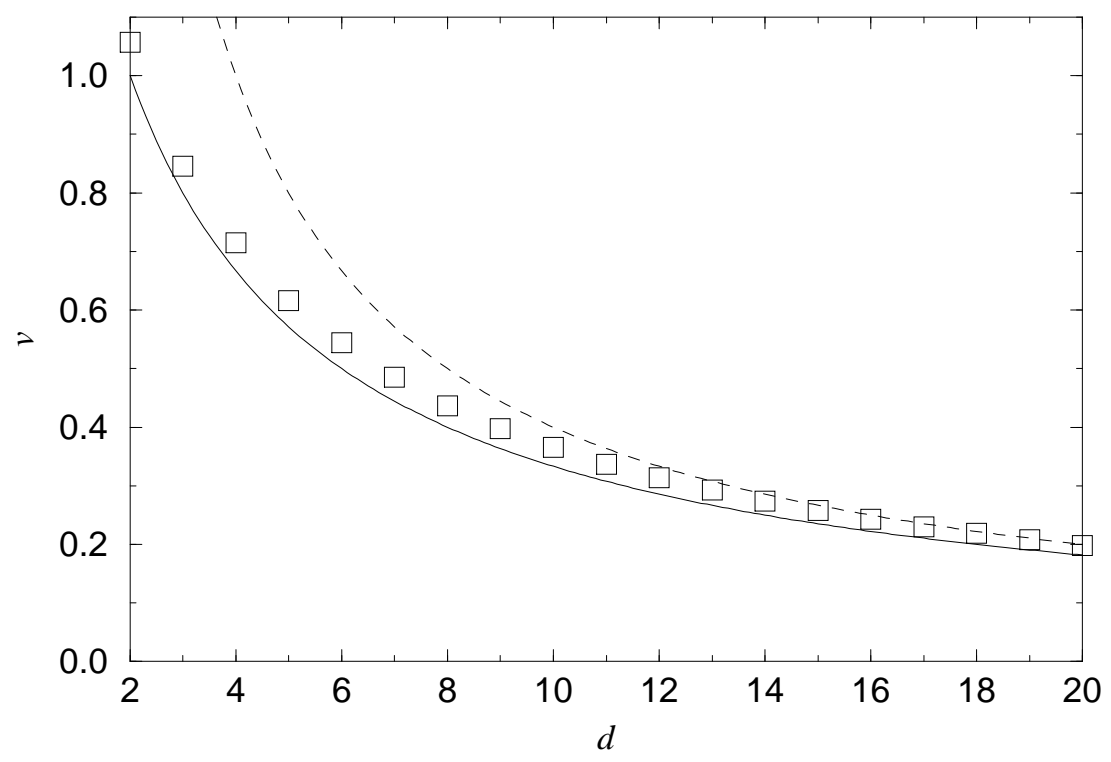

Figure 6.1: Extrapolation of the 2-loop results in $d$ and $\varepsilon$ for membranes $D=2$ in $d$ dimensions, using the expansion of $\nu^{*}(d+2)$ (squares). The solid line is the prediction made by Flory's approximation, the dashed line by the variational ansatz.

Let us now turn to the physically relevant case of membranes in three dimensions $(D=2, d=3)$. Our calculations predict an exponent $\nu^{*} \approx 0.85$ or equivalently a fractal dimension of $d_{\mathrm{f}} \approx 2.4$, which is in agreement with those experiments and simulations which find a fractal phase. However, this is still under debate, and a lot of evidence has been collected that the flat phase is generic. (For a more detailed discussion of this problem, see the review [7].)

\section{Outlook}

In these lecture notes, we have only been able to discuss the simplest applications of non-local field theories. Much more has been achieved during the last years.

First of all, it has been demonstrated that the dynamics, described by a Langevin-equation

$$
\frac{\mathrm{d}}{\mathrm{d} t} r(x, t)=-\frac{\partial \mathcal{H}[r]}{\partial r(x, t)}+\zeta(x, t), \quad\left\langle\zeta(x, t) \zeta\left(x^{\prime}, t^{\prime}\right)\right\rangle=\lambda \delta^{D}\left(x-x^{\prime}\right) \delta\left(t-t^{\prime}\right)
$$

also leads to a renormalizable field theory [11], where the dynamic exponent $z$, defined by the decay of the autocorrelation function $\left\langle\left[r(x, t)-r\left(x, t^{\prime}\right)\right]^{2}\right\rangle \sim\left|t-t^{\prime}\right|^{2 / z}$ is given by $z=2+D / \nu^{*}=2+d_{\mathrm{f}}$, where $d_{f}=D / \nu^{*}$ is the fractal dimension of the membrane. This result had been suggested long-time ago by DeGennes [12] for polymers and by Kardar et al. for membranes [13], but only with the methods discussed above, a proof of that conjectures could be given [11]. When hydrodynamics is included, the dynamical exponent $z$ changes to $z=d$ [14].

Interestingly, also disorder can be treated via the same methods, since averaging over disorder leads to interactions very similar to self-avoidance. The above methods have been applied to the motion of a polymer or membrane in a static disordered force field with both potential and non-conserved parts - leading to new universal physics $[15,16]$.

Also the advection of a polymer in a turbulent flow has been analyzed [17], paralling the discusion of the passive advection of particles, which in the turbulence community is known as the passive scalar problem.

Another interesting generalization is to anisotropically tethered membranes [18-20]. In these models, the membrane is more rigid in one direction, forming a tubular phase.

One of the most useful tricks for self-avoiding polymers is the mapping onto a massive scalar field-theory, i.e. a $\phi^{4}$-theory [21]. In the limit, where the number of compoents $n \rightarrow 0$, results for self-avoiding polymers are obtained. Stated differently, $\phi^{4}$-theory is a generalization of self-avoiding polymers. Another generalization has been discussed above: The generalization to membranes with internal dimension $D \neq 1$. The question arizes, whether a common generalization of both the $\phi^{4}$-theory and polymerized membranes is possible. Such a model has indeed been constructed [22,23], and leads to interesting new physics. 
Another still open question is the analysis of the spectrum of subdominant operators [24]. This may give a hint of why polymerized membranes are generically seen flat in simulations.

\section{Acknowledgements}

It is a pleasure to thank the organizers of the workshop at Heidelberg, Hans Jürgen Pirner and Franz Wegner, for the opportunity to give these lectures. I wish the honoree of this volume 60 more years of fruitful scientific work.

\section{References}

[1] A. Elsgaeter, B.T. Stokke, A. Mikkelsen and D. Branton, The molecular basis of erythrocyte shape, Science 234 (1986) 1217.

[2] C.F. Schmidt, K. Svoboda, N. Lei, I.B. Petsche, L.E. Berman, C.R. Safinya and G.S. Grest, Existence of a flat phase in red cell membrane skeletons, Science 259 (1993) 952.

[3] T. Hwa, E. Kokufuta and T. Tanaka, Conformation of graphite oxide membranes in solution, Phys. Rev. A 44 (1991) 2235.

[4] M.S. Spector, E. Naranjo, S. Chiruvolu and J.A. Zasadzinski, Conformations of a tethered membrane: Crumpling in graphitic oxide?, Phys. Rev. Lett. 73 (1994) 2867-2870.

[5] J.A. Aronovitz and T.C. Lubensky, $\varepsilon$-expansion for self-avoiding tethered surfaces of fractional dimension, Europhys. Lett. 4 (1987) 395-401.

[6] M. Kardar and D.R. Nelson, $\varepsilon$ expansions for crumpled manifolds, Phys. Rev. Lett. 58 (1987) 1289 and $2280 \mathrm{E}$.

[7] K.J. Wiese, Polymerized membranes, a review, volume 19 of Phase Transitions and Critical Phenomena, Acadamic Press, London, 1999.

[8] F. David, B. Duplantier and E. Guitter, Renormalization and hyperscaling for self-avoiding manifold models, Phys. Rev. Lett. 72 (1994) 311.

[9] F. David, B. Duplantier and E. Guitter, Renormalization theory for the self-avoiding polymerized membranes, cond-mat/9702136 (1997).

[10] F. David and K.J. Wiese, Large orders for self-avoiding membranes, Nucl. Phys. B 535 (1998) 555-595.

[11] K.J. Wiese, Dynamics of selfavoiding tethered membranes I, model A dynamics (Rouse model), Eur. Phys. J. B 1 (1998) 269-272.

[12] P.G. De Gennes, Dynamics of entangled polymer solutions. I. The Rouse model, Macromolécules 9 (1976) 587.

[13] Y. Kantor, M. Kardar and D.R. Nelson, Tethered surfaces: Statics and dynamics, Phys. Rev. A 35 (1987) 3056-3071.

[14] K.J. Wiese, Dynamics of selfavoiding tethered membranes II, inclusion of hydrodynamic interactions (Zimm model), Eur. Phys. J. B 1 (1998) 273-276.

[15] P. Le Doussal and K.J. Wiese, Glassy trapping of elastic manifolds in nonpotential static random flows, Phys. Rev. Lett. 80 (1998) 2362.

[16] K.J. Wiese and P. Le Doussal, Polymers and manifolds in static random flows: a RG study, Nucl. Phys. B 552 (1999) 529-598.

[17] K.J. Wiese, The passive polymer problem, chao-dyn/9911005 (1999).

[18] M. Bowick, M. Falcioni and G. Thorleifsson, Numerical observation of a tubular phase in anisotropic membranes, Phys. Rev. Lett. 79 (1997) 885.

[19] M. Bowick and E. Guitter, Effects of self-avoidance on the tubular phase of anisotropic membranes, Phys. Rev. E 56 (1997) 7023.

[20] M. Bowick and A. Travesset, The tubular phase of self-avoiding anisotropic crystalline membranes, Phys. Rev. E59 (1999) 5659.

[21] P.G. De Gennes, Exponents for the excluded volume problem as derived by the Wilson method, Phys. Lett. A 38 (1972) 339-340.

[22] K.J. Wiese and M. Kardar, Generalizing the $O(N)$-field theory to $N$-colored manifolds of arbitrary internal dimension D, Nucl. Phys. B 528 (1998) 469-522. 
[23] K.J. Wiese and M. Kardar, A geometric generalization of field theory to manifolds of arbitrary dimension, Eur. Phys. J. B 7 (1998) 187-190.

[24] K.J. Wiese and M. Shpot, The spectrum of irrelevant operators for self-avoiding membranes, in preparation.

[25] F. David, B. Duplantier and E. Guitter, Renormalization of crumpled manifolds, Phys. Rev. Lett. 70 (1993) 2205.

[26] F. David, B. Duplantier and E. Guitter, Renormalization theory for interacting crumpled manifolds, Nucl. Phys. B 394 (1993) 555-664.

[27] B. Duplantier, Nonuniversality in crumpled manifolds, Phys. Rev. Lett. 58 (1987) 2733-2737.

[28] B. Duplantier, T. Hwa and M. Kardar, Self-avoiding crumpled manifolds: Perturbative analysis and renormalizability, Phys. Rev. Lett. 64 (1990) 2022. 